\title{
PATHOGENETIC MECHANISM OF STENOSIS IN THE INTRAMURAL CORONARY ARTERY
}

\author{
Kazuo OHNishi, M.D.
}

\begin{abstract}
In order to clarify the pathogenetic mechanism of stenosis in the intramural coronary branch, morphological changes were examined by postmortem coronary angiography and determination of the Coronary Stenosis Index of the Myocardium (CSIM) were made in hearts obtained by autopsy from 10 patients with hypertension, 15 with normotensive myocardial infarction, 25 with hypertensive myocardial infarction, 6 with mitral valvular disease, 7 with aortic stenosis and 14 control subjects.

CSIM was significantly higher in patients with hypertension, infarction with hypertension and aortic stenosis than in other three groups, indicating higher incidence of stenosis in those with a left ventricular pressure load. There was no specific correlation between the degree of stenosis of the epicardial coronary artery and that of the intramural coronary branches supplied by the former in any groups examined. Major pathological changes of the intramural coronary branches were muscular hypertrophy of media and proliferation of internal elastic lamella, both observed with a high incidence in vessels with high CSIM values.

These findings suggest that intramural pressure which exerted extravascular pressure played an important role in the pathogenetic mechanisms of stenotic changes in the intramural coronary branches.
\end{abstract}

$\mathbf{A}$

MONG many variables affecting intramyocardial blood flow, mechanical factors have been reported to be important ${ }^{1,2}$ In addition, stenotic changes of the intramural branches of the coronary artery are thought to exercise great influence upon intramural blood flow.

Although there are many studies on the morphological aspects of intramural coronary arteries, ${ }^{3-6}$ only few reports ${ }^{7}$ have examined the correlation between the stenotic changes of the intramural branches and the mechanical factors existing in the ventricular wall.

\footnotetext{
Key Words:

Intramyocardial coronary stenosis

Epicardial coronary stenosis

Intramyocardial pressure

Coronary perfusion pressure

Left ventricular hypertrophy
}

The purpose of this study is to detect stenotic changes in the intramural branches of the coronary artery by means of microangiography, histometric methods and pathological observation, and to correlate them with intraventricular pressure and aortic pressure. Thus the relation ship of the pathogenetic mechanism of the stenotic changes and mechanical factors such as coronary perfusion pressure, intramyocardial pressure and vascular tension can be clarified.

\section{MATERIALS AND METHODS}

\section{Subjects}

Hearts obtained by autopsy from 77 patients without diabetes mellitus at Kobe Rosai Hospital were studied. Cases were divided into the following six groups based upon the status of the left ventricular pressure overload.

(Received August 2, 1985; accepted April 11, 1986)

Department of Internal Medicine, Kobe Rosai Hospital, Kobe, Japan

Mailing address: Kazuo Ohnishi, M.D., Department of Internal Medicine, Kobe Rosai Hospital, 4-1-23, Kagoike-dori, Chuo-ku, Kobe 651, Japan 


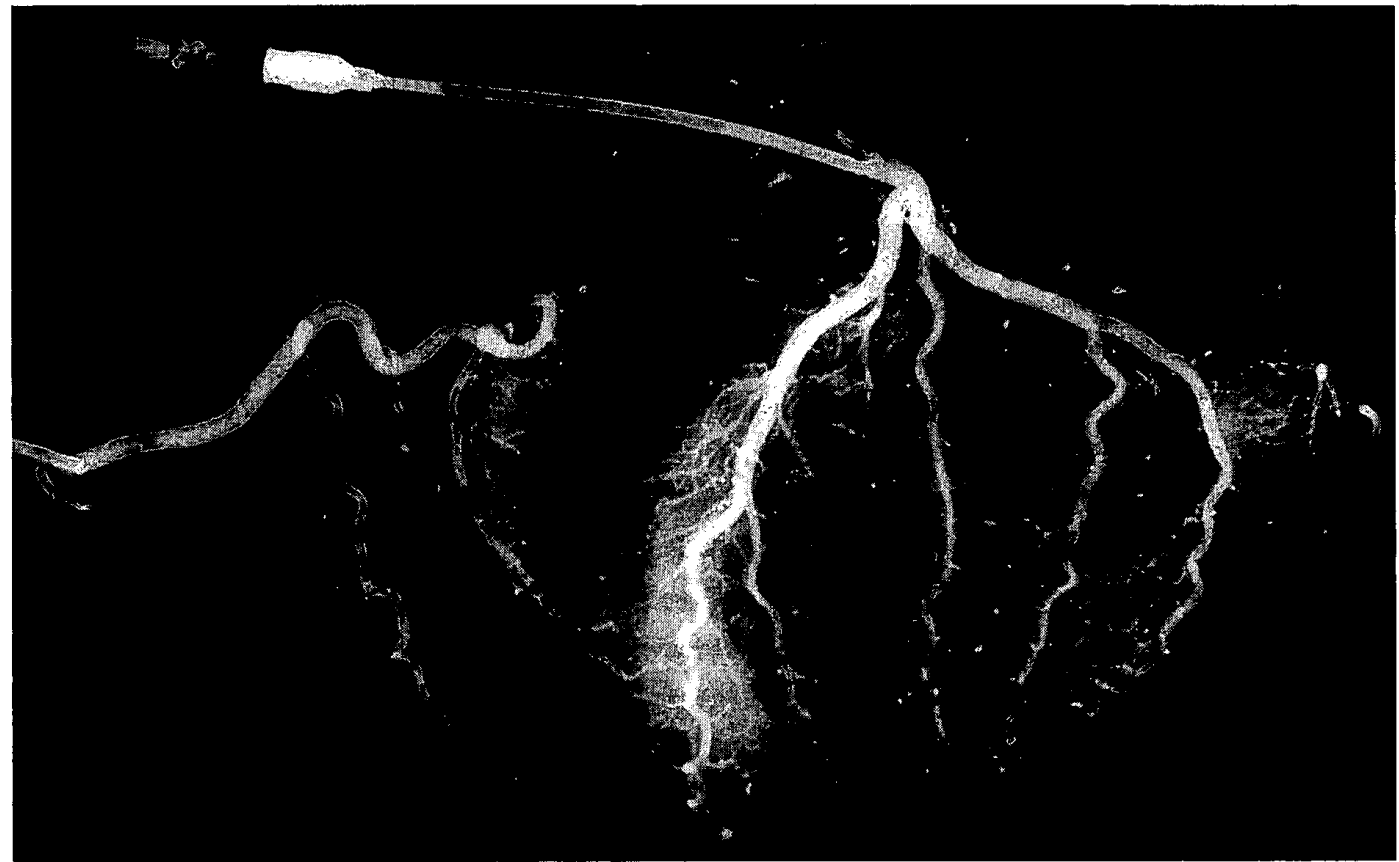

Fig.1. Postmortem coronary angiogram of Rodriguez-Reiner's unrolled heart.

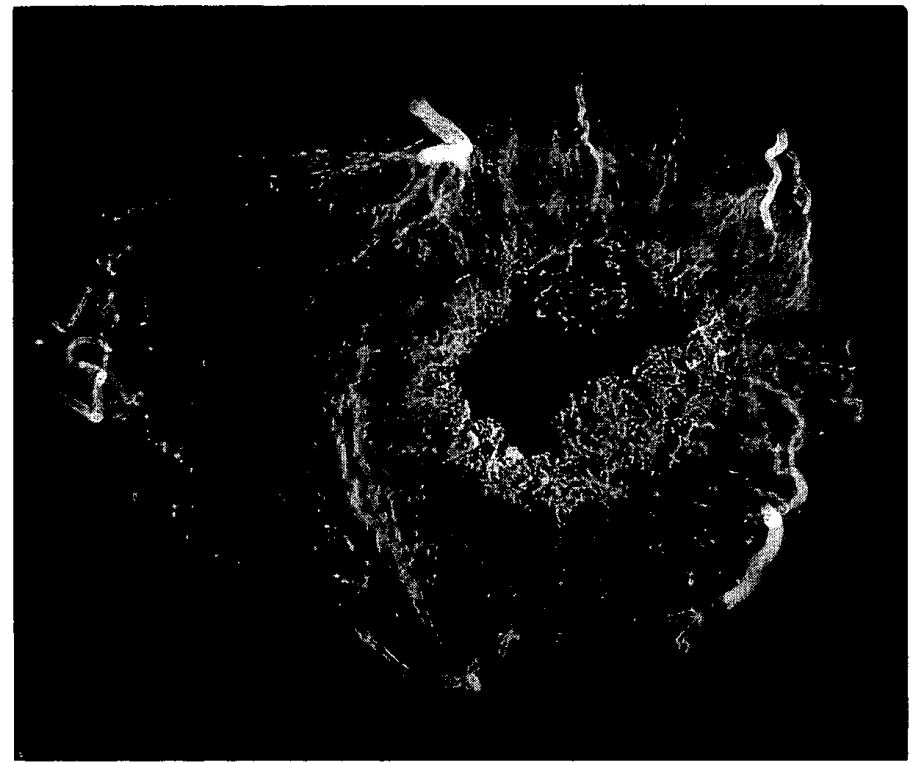

Fig.2. Postmorten coronary angiogram of cross-sectional view in a case with hypertension.

1) Control group was composed of 14 patients (male 7, female 7) with no evidence of heart diseases either on clinical examinations or at autopsy. In addition, none had a history of hypertension. Ages ranged from 43 to 85 years with a mean of $63.0 \pm 11.5$.

2) Hypertension group was composed of 10 patients (male 6, female 4) who had obvious histories of hypertension diagnosed by WHO criteria over 3 years, who died of cerebral hemorrhage or renal failure, and who showed concentric left ventricular hypertrophy on autopsy. Ages ranged from 43 to 72 years with a mean of $61.7 \pm 9.8$.

3) Myocardial infarction without hypertension (Infarct-N) group was composed of 15 patients (male 12, female 3) with histories of obvious clinical episodes and autopsy findings of myocardial infarction but without a history of hypertension. Ages ranged from 36 to 82 years with a mean of $62.3 \pm 11.2$.

4) Myocardial infarction with hypertension Japanese Circulation Journal Vol. so, October 1986 

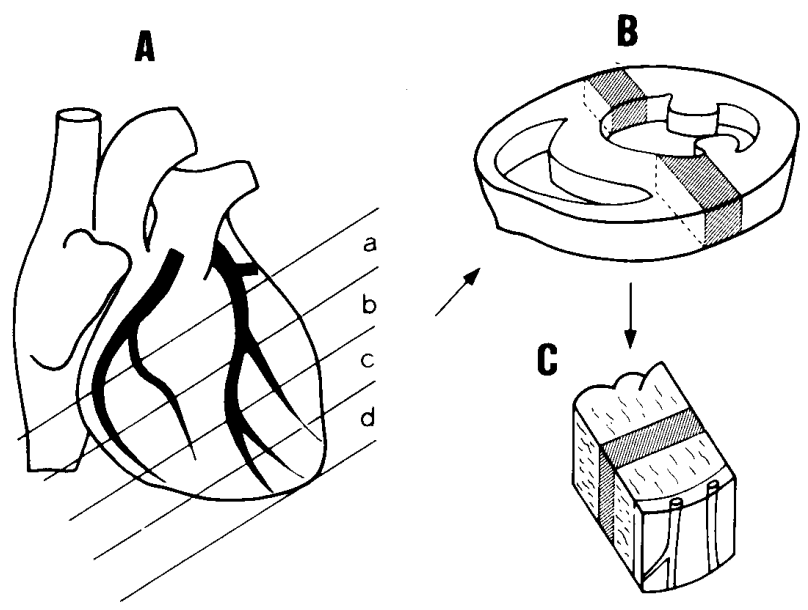

Fig.3. Scheme of tissue preparation for microscopic examination.

(Infarct-H) group was composed of 25 patients (male 17, female 8) with definite clinical histories and autopsy findings of myocardial infarction and with obvious histories of hypertension of over 3 years. Ages ranged from 46 to 81 years with a mean of $66.3 \pm 8.5$.

5) Mitral valvular disease (Valvular-M) group was composed of 6 patients (male 2, female 4) who were diagnosed on clinical examination as having either mitral stenosis or mitral stenoinsufficiency and who had neither a change in the aortic valve nor left ventricular hypertrophy on autopsy. Ages ranged from 57 to 81 years with a mean of $68.3 \pm 10.1$.

6) Aortic valvular stenosis (Valvular-A) group was composed of 7 patients (male 3, female 4) who were diagnosed on clinical examination as having aortic valvular stenosis and who showed adhesion and thickening of the aortic cusps and marked left ventricular hypertrophy on autopsy. Ages ranged from 49 to 88 years with a mean of $69.3 \pm 13.2$.

\section{Postmortem Coronary Angiography}

A mixture of $50 \%$ microbarium sulfate (Baritogen sol ${ }^{\circledR}$ and $10 \%$ gelatin solution was injected under a pressure of $100 \mathrm{mmHg}$ for five minutes into the right and left coronary arteries of the heart excised at autopsy. X-ray pictures of the horizontal cross-section of the ventricle and the heart unrolled with the method of Rodriguez-Reiner ${ }^{8}$ were taken using an ultrasoft X-ray apparatus (Softex Type CMB). (Fig.1 and 2)

\section{Preparation of Tissue Specimens}

The left ventricles were sectioned horizontally

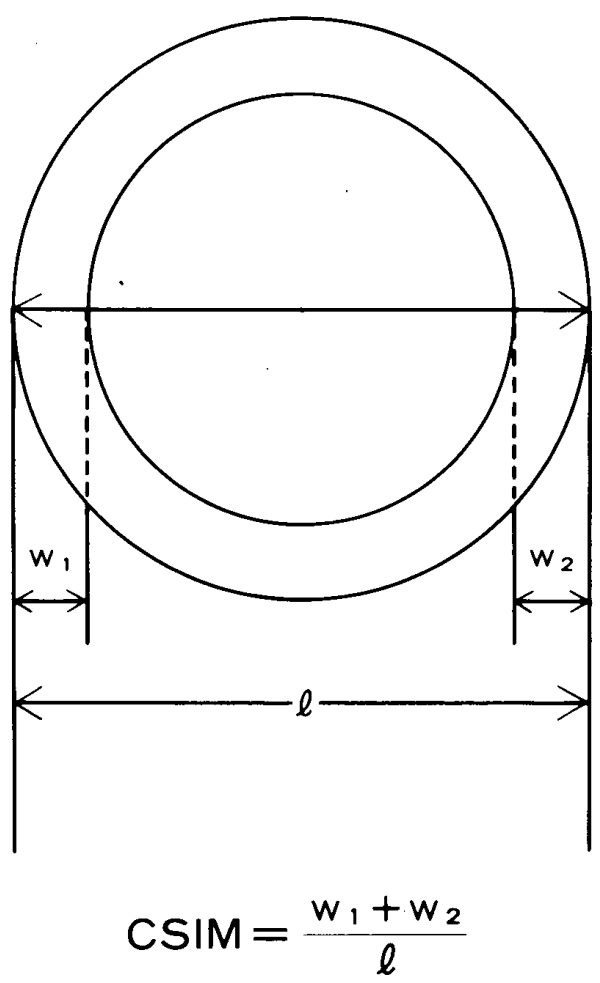

Fig.4. Formula for calculating the Coronary Stenosis Index of the myocardium (CSIM).

into four portions between the base and the apex as shown in Fig. 3-A. From slice C, each block of the myocardium was excised from the anterior- and posterior-free walls. In high grade scar tissues caused by myocardial infarction, the portion surrounding the scar was excised. In excising the myocardial block, its source of the epicardial coronary artery was identified, referring to the angiographic picture of the horizontal cross-section. Tissue slices that were parallel to the epicardial surface and vertical to the intramural branch of the coronary artery were prepared from the center of the excised myocardial block (Fig. 3-C) and Hematoxylin. Eosin, Elastica van Gieson and Azan stains were performed.

\section{Determination of the Degree of Stenosis}

The degree of stenosis of the epicardial coronary artery was calculated from the inner diameter of the most stenotic portion of the three major branches using the postmortem coronary angiogram. Stenosis was also confirmed macroscopically by opening the most stenotic portion of the coronary artery. The narrowing of the diameter was classified into the following four groups: (1) less than 50\%, (2) 50 75\%, (3) $75 \sim 99 \%$ and (4) complete occlusion. For 

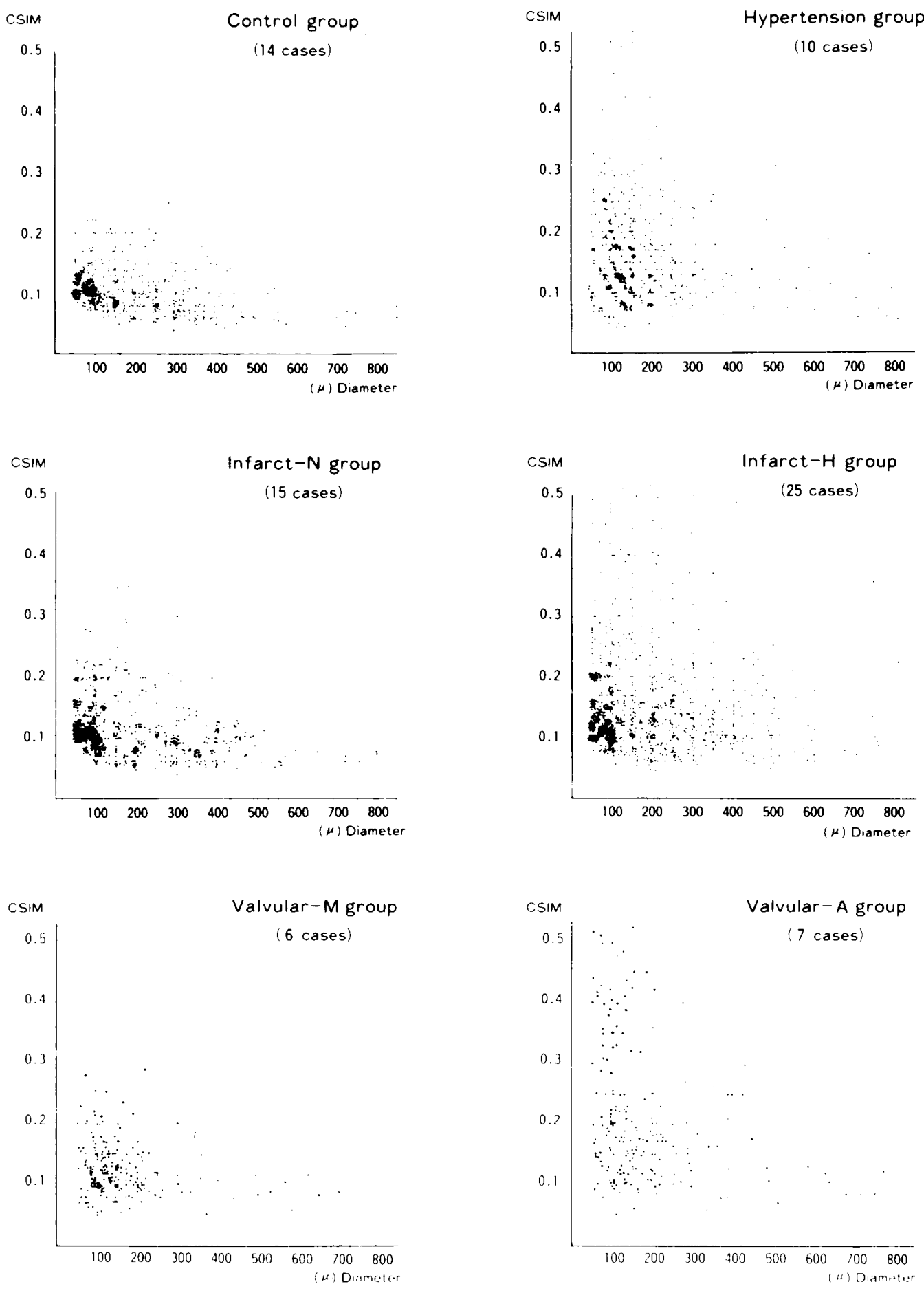

Fig.5. Relationship between diameter of the intramual branch and CISM.

measurement of the intramural coronary arteries, vessels not fully filled with contrast medium and those in the fibrotic scarred tissue were excluded. Small arteries with an outer diameter of 50 microns or greater and with a round cross-section were selected at random and measured. Vessels with a slightly oblong cross-section were regarded as having a round cross section with a diameter equal to its shorter diameter. Consequently, 25 to 51 (mean 39.7) vessels were measured for one tissue specimen. The outer diameter excluding the adventitia (1) and the thickness of the vascular walls at the both ends of the diameter $\left(\mathrm{w}_{1}, \mathrm{w}_{2}\right)$ of the intramural branch of the coronary artery were measured with a microscopic micrometer. 

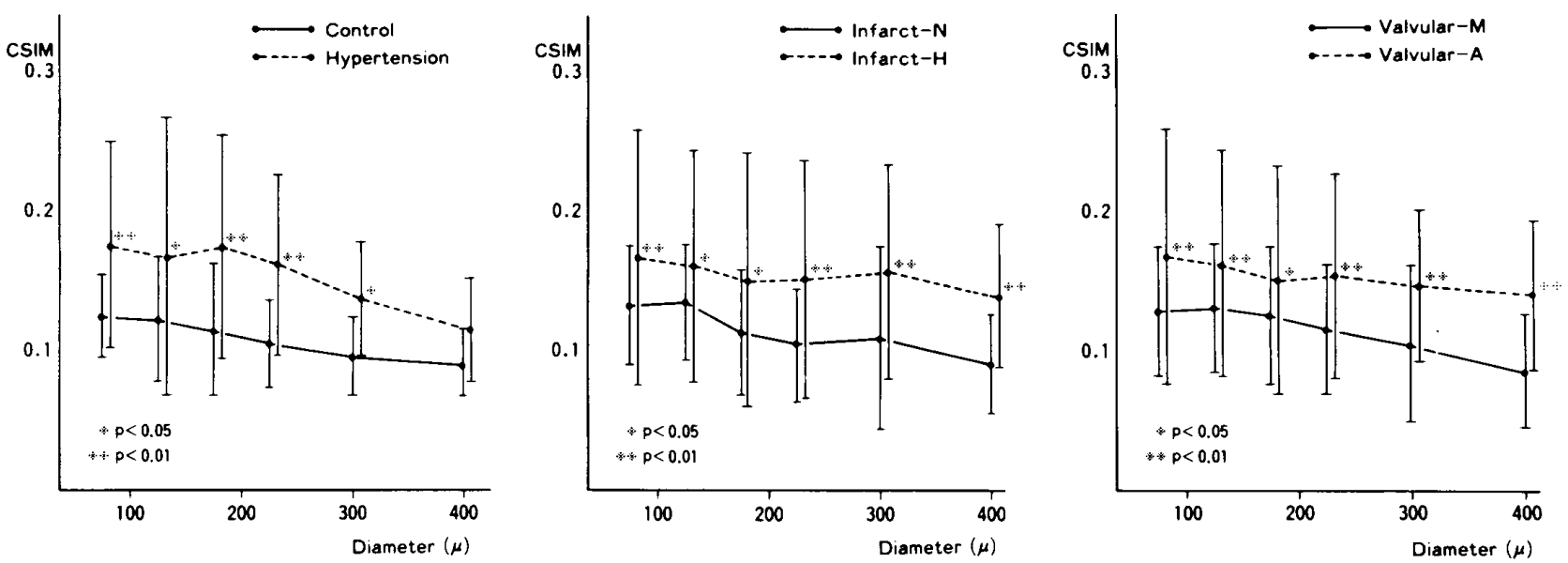

Fig.6. Mean CSIM and diameter of the intramural coronary branch.

TABLE I FREQUENCY OF INTRAMURAL BRANCHES WITH CSIM OVER MEAN + 2 S.D. OF CONTROL GROUP

\begin{tabular}{lcccc}
\hline \hline \multirow{2}{*}{ Group } & & \multicolumn{4}{c}{ Diameter of Intramural Branches $(\mu)$} & \\
\cline { 2 - 5 } & $50 \sim 99$ & $100 \sim 299$ & over 300 & Total \\
\hline Control & $13 / 223(5.8 \%)$ & $9 / 223(4.0 \%)$ & $2 / 95(2.1 \%)$ & $24 / 541(4.4 \%)$ \\
Hypertension & $37 / 109(33.9 \%)$ & $72 / 259(27.8 \%)$ & $13 / 57(22.8 \%)$ & $122 / 425(28.7 \%)$ \\
Infarct- $N$ & $31 / 235(13.2 \%)$ & $27 / 236(11.4 \%)$ & $12 / 130(9.2 \%)$ & $70 / 601(11.6 \%)$ \\
Infarct-H & $111 / 399(27.8 \%)$ & $110 / 391(28.1 \%)$ & $47 / 160(29.4 \%)$ & $268 / 950(28.2 \%)$ \\
Valvular $-M$ & $9 / 85(10.5 \%)$ & $12 / 163(7.4 \%)$ & $3 / 29(10.3 \%)$ & $24 / 277(8.6 \%)$ \\
Valvular $-A$ & $45 / 75(60.0 \%)$ & $51 / 132(38.6 \%)$ & $13 / 28(46.4 \%)$ & $109 / 235(46.4 \%)$ \\
\hline
\end{tabular}

The degree of stenosis of the branch was expressed as a ratio of the thickness of the vascular walls to the outer diameter of the vessel $\left(\left(w_{1}+w_{2}\right) / 1\right)$. This ratio was termed as the Coronary Stenosis Index of the Myocardium (CSIM) (Fig. 4).

Pathological changes of the intramural coronary artery were also observed and the incidence of histological changes of the intima and the media which affected CSIM values were also obtained. The results were analyzed statistically with Student's t-test and the differences were judged as significant when $p$ values were less than 0.05 .

\section{RESULTS}

Coronary Stenosis Index of the Myocardium (CSIM)

1) Relationship between CSIM and the outer vascular diameter.
In the Control group, CSIM was in most cases 0.2 or less and showed a tendency to decrease as the outer vascular diameter increased (Fig. 5). CSIM in this group showed a value of $0.124 \pm 0.033,0.122 \pm 0.046,0.115 \pm 0.047$ and $0.105 \pm 0.031$ for vessels with a diameter of $50 \sim 99, \quad 100 \sim 149, \quad 150 \sim 199$ and 200 250 microns, respectively. The outer diameter and CSIM showed a very similar relationship in each group of the Control, Infarct-N and Valvular-M groups (Fig. 5). In contrast, CSIM showed wide variation from less than 0.1 to more than 0.5 and many cases indicated high values in the Hypertension, Infarct-H and Valvular-A groups. The distribution of CSIM was similar to each other among these three groups.

The Hypertension group, the Infarct-H group and the Valvular-A group had significantly higher CSIM values than the Control group, the Infarct-N group and Valvular-M group, respectively, for all ranges of outer diameter of the 

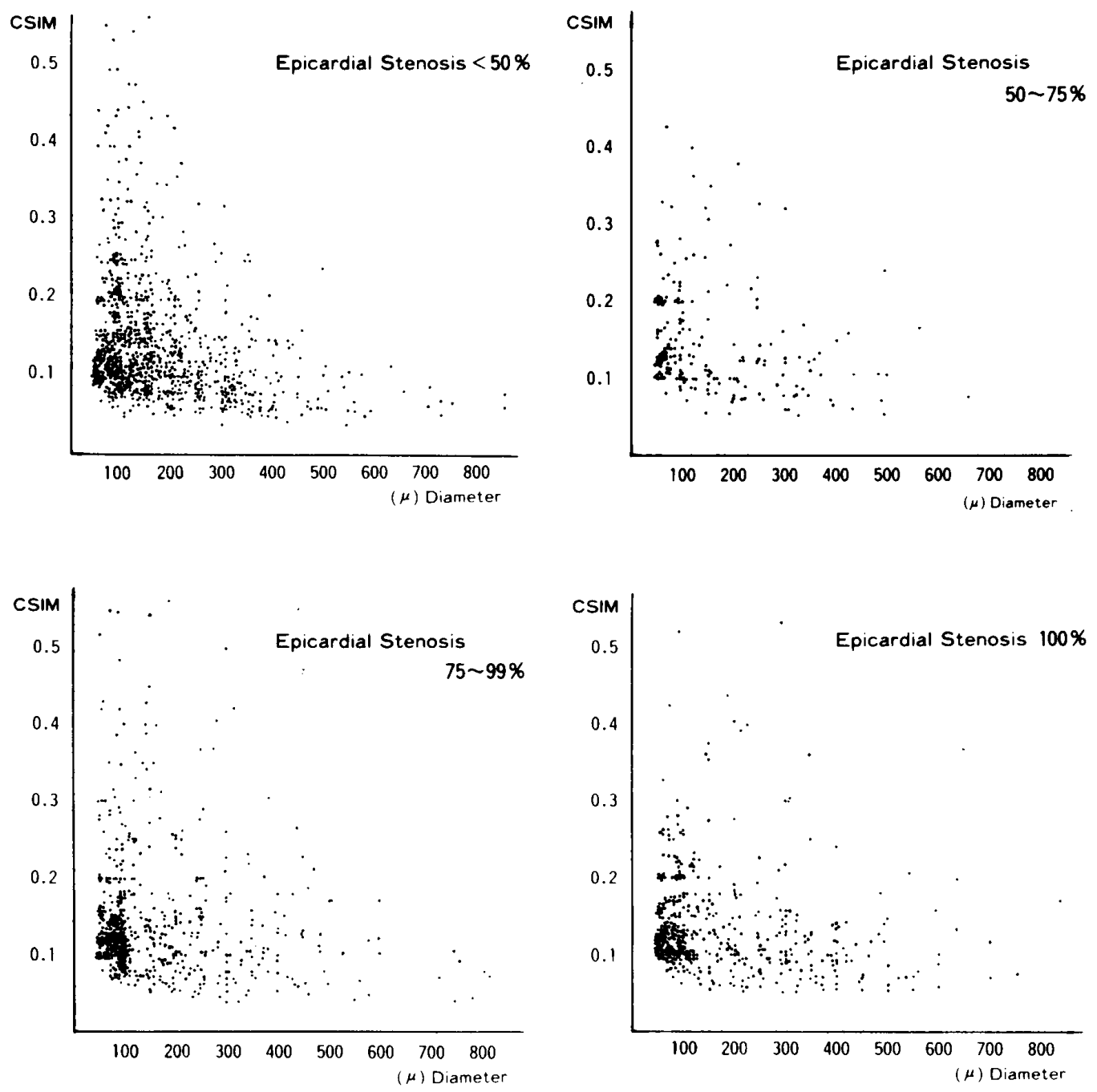

Fig.7. Relationship between diameter of intramural branch and CSIM in various degrees of epicardial coronary stenosis.

vessels $(\mathrm{p}<0.01$ or $\mathrm{p}<0.05$ ) (Fig. 6). There was no significant difference in CSIM among the Control, Infarct-N and Valvular-M groups, and also there was no significant difference in CSIM between the Hypertension, Infarct- $\mathrm{H}$ and Valvular-A groups for all ranges of outer diameter of the vessels $(\mathrm{p}<0.01$ or $\mathrm{p}<0.05)$.

2) Incidence of severely stenotic intramural branches.

The value of the upper limit of CSIM (mean CSIM + 2S.D.) in the Control group was 0.19, 0.19 and 0.16 for 50 99, 100 299 and over 300 microns of the outer diameter, respectively. Vessels with CSIM higher than these values were regarded as severely stenotic intramural branch and their incidence for each group was compared (Table I).

The incidence in the Control, Infarct- $\mathrm{N}$ and Valvular-M group was 4.4, 11.6 and $8.6 \%$, respectively and these values were relatively low. In contrast, severely stenotic intramural branches were frequently observed in the Hypertension, Infarct-H and Valvular-A groups with an incidence of $28.7,28.2$ and $46.4 \%$, respectively. The incidence was particularly high in the Valvular-A group. In the Hypertension group and the Valvular-A group, severely stenotic intramural branches tended to be present in those with an outer diameter of 50 99 microns than those with other diameters. In other groups, however, there was no consistent tendency in the incidence of severely stenotic branches associated with the outer diameter.

3) Relationship between epicardial coronary arterial stenosis and CSIM.

First, the relationship between the degree of epicardial coronary arterial stenosis and the CSIM value of the intramural branch supplied 


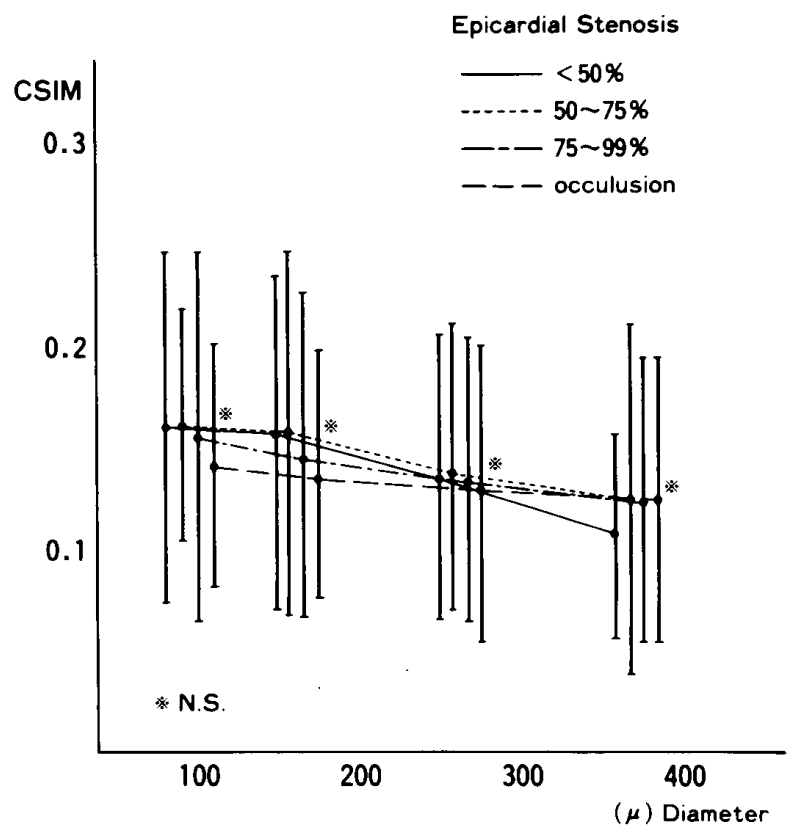

Fig.8. Mean CSIM and diameter of intramural branch in various degrees of epicardial coronary stenosis. by the same epicardial coronary artery was examined for all groups combined as a single unit. In Fig. 7 the correlograms between CSIM and the outer diameter of the intramural branch for various degrees of stenosis of the epicardial coronary artery supplying the intramural branch are shown. CSIM and the outer diameter of the intramural branch showed similar correlograms independent on the degree of stenosis of the epicardial coronary artery. There was no significant difference in the mean CSIM of intramural branches regardless of their diameter among cases with various degrees of stenosis of epicardial coronary arteries (Fig. 8).

Next, the relationship between the degree of stenosis of the epicardial coronary artery and the incidence of severely stenotic intramural branches was examined for each group. This was also done for measures of the various outer diameters of the intramural branch. In either case, these was no specific relationship between

TABLE II FREQUENCY OF INTRAMURAL BRANCHES WITH CSIM OVER MEAN + 2S.D. IN THE DISTAL AREA TO EPICARDIAL STENOSIS

\begin{tabular}{|c|c|c|c|c|c|}
\hline \multirow{2}{*}{ Group } & \multirow{2}{*}{$\begin{array}{l}\text { Diameter of } \\
\text { Intramural } \\
\text { Branch }(\mu)\end{array}$} & \multicolumn{4}{|c|}{ Stenosis of Epicardial Branch (\%) } \\
\hline & & less than $50 \%$ & $50 \sim 75 \%$ & $75 \sim 99 \%$ & occlusion \\
\hline \multirow[t]{3}{*}{ Control } & $50 \sim 99$ & $13 / 223(5.8)$ & 0 & 0 & 0 \\
\hline & $100 \sim 299$ & $9 / 223(4.0)$ & 0 & 0 & 0 \\
\hline & over 300 & $2 / 95(2.1)$ & 0 & 0 & 0 \\
\hline \multirow[t]{3}{*}{ Hypertension } & $50 \sim 99$ & $29 / 80 \quad(36.3)$ & 0 & $4 / 18 \quad(22.2)$ & $4 / 11 \quad(36.4)$ \\
\hline & $100 \sim 299$ & $56 / 172(32.9)$ & 0 & $10 / 73(13.7)$ & $6 / 14 \quad(42.9)$ \\
\hline & over 300 & $5 / 28 \quad(17.9)$ & 0 & $5 / 12 \quad(41.7)$ & $3 / 17 \quad(17.6)$ \\
\hline \multirow[t]{3}{*}{ Infarct- $N$} & $50 \sim 99$ & $3 / 45 \quad(6.7)$ & $3 / 30 \quad(10.0)$ & $4 / 52(7.7)$ & $21 / 108(19.4)$ \\
\hline & $100 \sim 299$ & $7 / 48 \quad(14.6)$ & $3 / 31$ (9.7) & $7 / 54 \quad(13.0)$ & $10 / 103(9.7)$ \\
\hline & over 300 & $3 / 22(13.6)$ & $2 / 11 \quad(18.2)$ & $4 / 38 \quad(10.5)$ & $3 / 59(5.1)$ \\
\hline \multirow[t]{3}{*}{ Infarct- $H$} & $50 \sim 99$ & $14 / 46(30.4)$ & $30 / 64$ (46.9) & $36 / 122(29.5)$ & $31 / 167(18.6)$ \\
\hline & $100 \sim 299$ & $13 / 49 \quad(26.5)$ & $21 / 58 \quad$ (36.2) & $41 / 146(28.1)$ & $35 / 138(25.4)$ \\
\hline & over 300 & $6 / 20 \quad(30.0)$ & $8 / 21(38.1)$ & $15 / 58 \quad(25.9)$ & $18 / 61$ \\
\hline \multirow[t]{3}{*}{ Valvular-M } & $50 \sim 99$ & $9 / 85 \quad(10.6)$ & 0 & 0 & 0 \\
\hline & $100 \sim 299$ & $12 / 163(7.4)$ & 0 & 0 & 0 \\
\hline & over 300 & $3 / 29 \quad(10.3)$ & 0 & 0 & 0 \\
\hline \multirow[t]{3}{*}{ Valvular- $A$} & $50 \sim 99$ & $32 / 52 \quad(61.5)$ & 0 & $13 / 23 \quad(56.5)$ & 0 \\
\hline & $100 \sim 299$ & $35 / 85 \quad(41.2)$ & 0 & $16 / 47(34.0)$ & 0 \\
\hline & over 300 & $8 / 18 \quad(44.4)$ & 0 & $5 / 10 \quad(50.0)$ & 0 \\
\hline
\end{tabular}



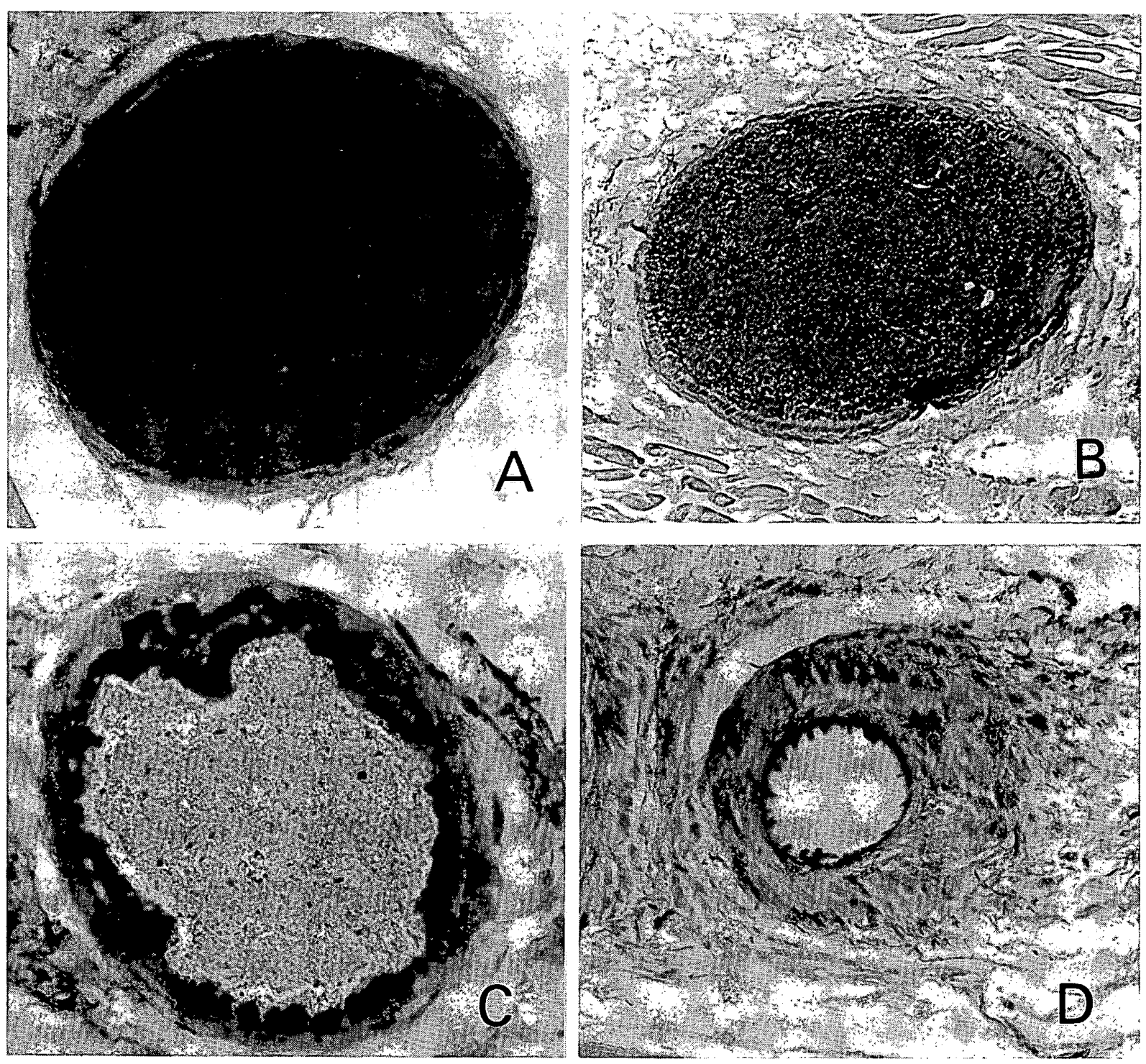

Fig.9. Typical histological changes in the intramural branch.

A: Normal. B: Thickening of the intima. C: Proliferation and multiplication of the elastica interna. D: Hypertrophy of the media.

the degree of stenosis of the epicardial coronary artery and the incidence of severely stenotic intramural branches (Table II). From the findings above, it was confirmed that there is no specific relationship between the stenosis of the epicardial coronary artery and the degree of stenosis of the intramural branch supplied by the former.

\section{Histopathological Changes of the Intramural Branch}

Major histological changes which resulted in stenosis of the intramural branch were thickening of the intima (Fig. 9-B), multiplication of the elastica interna due to its proliferation (Fig. 9-C) and muscular hypertrophy of the media (Fig. 9-D). The adventitia was loose with an ill-defined border with the surrounding tissue. Therefore, the adventitia was excluded in the calculation of CSIM and its pathological findings were not examined. The incidence of such histopathological findings was examined for each group of patients and for various outer diameters of the vessel (Table III).

Thickning of the intima was not observed at all in the Control group and was observed in a very low incidence in other groups.

Multiplication of the elastica interna in the Control group occurred in $0.9,1.3$ and $5.3 \%$ of the vessels with a diameter of 50 99, 100 299 and 300 microns or greater, respectively. In the Infarct-N group and the Valvular-M group also, incidence of multiplication of the elastica interna was low, which was a similar finding in the 
TABLE III DIAMETER OF THE INTRAMURAL BRANCHES AND THEIR HISTOLOGICAL CHANGES

\begin{tabular}{|c|c|c|c|c|c|}
\hline \multirow[b]{2}{*}{ Group } & \multirow{2}{*}{$\begin{array}{c}\text { Diameter of } \\
\text { Vessels } \\
(\mu)\end{array}$} & \multirow[b]{2}{*}{$\begin{array}{l}\text { Total No. } \\
\text { of Vessels }\end{array}$} & \multicolumn{3}{|c|}{ Pathological Findings (\%) } \\
\hline & & & $\begin{array}{l}\text { Thickening } \\
\text { of Intima }\end{array}$ & $\begin{array}{c}\text { Multiplication } \\
\text { of Elastica } \\
\text { interna }\end{array}$ & $\begin{array}{l}\text { Hypertrophy } \\
\text { of Media }\end{array}$ \\
\hline \multirow[t]{3}{*}{ Control } & $50 \sim 99$ & 223 & 0 & $2(0.9)$ & $12(5.4)$ \\
\hline & $100 \sim 299$ & 223 & 0 & $3(1.3)$ & $16(7.2)$ \\
\hline & over 300 & 95 & 0 & $5(5.3)$ & $3(3.2)$ \\
\hline \multirow[t]{3}{*}{ Hypertension } & $50 \sim 99$ & 109 & $1(0.9)$ & $20(18.3)$ & $25(22.9)$ \\
\hline & $100 \sim 299$ & 259 & $4(1.5)$ & $57(22.0)$ & $101(39.0)$ \\
\hline & over 300 & 57 & $1(1.8)$ & $15(26.3)$ & $12(21.1)$ \\
\hline \multirow[t]{3}{*}{ Infarct- $N$} & $50 \sim 99$ & 235 & $1(0.4)$ & $2(0.8)$ & $16(7.1)$ \\
\hline & $100 \sim 299$ & 236 & $6(2.6)$ & $8(3.5)$ & $43(19.0)$ \\
\hline & over 300 & 130 & $2(1.6)$ & $8(6.3)$ & $19(15.1)$ \\
\hline \multirow[t]{3}{*}{ Infarct- $H$} & $50 \sim 99$ & 399 & 0 & $27(6.7)$ & $114(28.6)$ \\
\hline & $100 \sim 299$ & 391 & $8(2.0)$ & $64(16.4)$ & $179(44.0)$ \\
\hline & over 300 & 160 & $1(0.6)$ & $27(17.3)$ & $47(30.1)$ \\
\hline \multirow[t]{3}{*}{ Valvular-M } & $50 \sim 99$ & 85 & 0 & $1(1.2)$ & $4(4.7)$ \\
\hline & $100 \sim 299$ & 163 & 0 & $8(4.9)$ & $13(8.0)$ \\
\hline & over 300 & 29 & $1(3.4)$ & $1(3.4)$ & $2(6.9)$ \\
\hline \multirow[t]{3}{*}{ Valvular- $A$} & $50 \sim 99$ & 75 & 0 & $8(10.7)$ & $31(41.3)$ \\
\hline & $100 \sim 299$ & - 132 & $2(1.5)$ & $28(21.2)$ & $60(45.5)$ \\
\hline & over 300 & 28 & $1(3.6)$ & $6(21.4)$ & $12(42.9)$ \\
\hline \multirow{3}{*}{$\begin{array}{l}\text { Statistical Analysis in } \\
\text { Each Diameter of Vessels }\end{array}$} & \multicolumn{2}{|c|}{ Control vs Hypertension } & $N . S$. & $p<0.01$ & $p<0.01$ \\
\hline & \multicolumn{2}{|c|}{ Infarct- $N$ vs infarct- $H$} & N.S. & $p<0.01$ & $p<0.01$ \\
\hline & \multicolumn{2}{|c|}{ Valvular-M vs Valvular- $A$} & N.S. & $p<0.01$ & $p<0.01$ \\
\hline
\end{tabular}

Control group. In contrast, it was observed in significantly higher incidence in the Hypertension, Infact-H, and Valvular-A groups than in the Control, Infarct-N, and Valvuar-M groups for any outer diameter of the vessel $(p<0.01)$. In terms of the vascular diameter, multiplication of the elastica interna was observed in a slightly lower incidence in vessels with a diameter of 50 99 microns than those with a greater diameter.

Hypertrophy of the media in the Control group was observed in $5.4,7.2$ and $3.2 \%$ of the vessels with an outer diameter of 50 99, $100 \sim 299$ and 300 microns or greater, respectively. In the Valvular-M group also, it was seen with a similar incidence. In contrast, it occurred with significantly higher incidence in the Hypertension, Infarct-H and Valvular-A groups than in the Control group for any outer diameter of the vessel $(p<0.01)$. In the Infarct-N group, hypertrophy of the media was observed in 7.1 and $19.0 \%$ of the vessels with an outer diameter of 50 99 and 100 299 microns. The incidence was slightly higher in the Infarct-N group than in the Control group, but significantly lower than in the Hypertension, Infarct-H, and Valvular-A groups $(p<0.01)$. The incidence of hypertrophy of the media for various vascular diameters tended to be slightly higher in the vessels with a diameter of 100 299 microns than in those with other diameters in all the groups. 


\section{DISCUSSION}

Among many mechanical factors that affect morphological changes of the intramural branches of the coronary arteries, coronary perfusion pressure, intramyocardial pressure and vascular tension are important. In this study, cases of myocardial infarction (i.e. those with epicardial coronary stenosis) were compared with cases without epicardial stenosis in order to examine whether the changes of coronary perfusion pressure affect the morphological changes of the intramural coronary branches. Also, patients with hypertension and those of aortic valvular stenosis (i.e. those with left ventricular pressure overload) were compared with those without left ventricular pressure overload in order to examine the effect of the elevation of left ventricular pressure on the intramural branches.

For a quantitative assessment of the degree of stenosis of the epicardial coronary artery and its intramural branches, postmortem coronary angiography under a constant pressure of $100 \mathrm{mmHg}$ was performed in all cases and measurement of vascular dimension was taken in stretched intramural branches. The ratio of the sum of the thickness of the vascular walls at the ends of the diameter to the outer diameter of the intramural branch excluding the adventitia was termed Coronary Stenosis Index of the Myocardium (CSIM). This index was used for assessing the degree of stenosis of the intramural branch of the coronary artery. Because, in the conventional histological method without postmortem coronary angiography, ${ }^{4-6}$ the intramural branches frequently do not show a round cross section due to the effect of formalin fixation and difference in the stage of myocardial contraction at the time of cardiac arrest, such a conventional method was not suitable for determination of the degree of stenosis. Since, in this study, vascular dimensions were restored to a more natural state without destruction of vascular wall structure than in uninjected postmortem material, more accurate CSIM values were obtained?

Regarding the relationship of CSIM with the aortic pressure, it was revealed that CSIM in the Hypertension group was significantly higher than in the Control group, suggesting that CSIM was related closely to hypertension. Furthermore, in the analysis of the cases of myocardial infarction with stenosis of the epicardial coronary artery, those with hypertension showed significantly higher CSIM values than those without hyper- tension, suggesting a close relationship between CSIM and hypertension. In some previous reports, there was no consensus as to a correlation between the stenosis of the intramural branch and hypertension. Such discrepancy of the results is regarded to be mainly due to the difference in the method of the studies employed. Since the vessels are collapsed using the ordinary histological method, such a small difference in the vascular wall thickness as represented by 0.05 of CSIM value revealed in the present study would not be detected. Also difference in the distensibility of the vascular wall caused by structual changes of the wall would not be found with the ordinary method.

Since coronary perfusion pressure is affected by the degree of stenosis of the epicardial coronary artery, the correlations between coronary perfusion pressure and stenotic changes of the intramural coronary branches were examined by comparing the degree of stenosis of the epicardial coronary artery with the intramural branches supplied by it. In the human heart, it has been shown that antegrade pressure is roughly proportional to the estimated degree of proxymal stenosis and the mean antegrade pressure through the stenosis of $95 \%$ or greater averaged only $45 \%$ of aortic pressure.13 Retrograde pressure is low, usually about one-third of the systemic pressure and almost "never over $30 \mathrm{mmHg}$. Retrograde pressure is relatively independent of the degree of proxymal stenosis or of angiographically demonstrable collateral circulation. ${ }^{13}$ Therefore, it is assumed that there is some correlation between the degree of stenosis of the epicardial coronary artery and CSIM values of the intramural branches, if stenotic changes of the intramural branches are affected by coronary perfusion pressure. From the results of the present study, however, no specific correlation was found between the degree of stenosis of the epicardial coronary artery and CSIM values of the intramural branches supplied by it in any of the groups examined with or without hypertension. This suggests that CSIM values are not affected by coronary perfusion pressure.

The relationship between the intramural pressure and stenotic changes of the intramural branches was examined to clarify the relationship between the intraventricular pressure of the heart, which is closely related to the intramural pressure ${ }^{14,15}$ and CSIM values. CSIM values were significantly higher in the Hypertension, Infarct-H and Valvular-A groups than in the 
remaining three groups. Moreover, the incidence of severely stenotic intramural branches in the Valvular-A group in which left ventricular pressure alone had been markedly elevated without elevation of aortic pressure, were higher than those in the Hypertension and Infarct-H groups. Consequently, it is suggested that intraventricular pressure or intramural pressure of the left ventricle is a more important factor affecting CSIM values than aortic pressure. However, left ventricular hypertrophy was present in all cases in Hypertension, Infarct-H and Valvular-A groups. The possibility that the elevation of the CSIM values might be induced by the left ventricular hypertrophy could not be denied.

In histological observations of the intramural branches, changes in the intima were scarcely observed in the intramural coronary arterial wall regardless of the left ventricular pressure load or stenosis of the epicardial coronary artery. In contrast, hypertrophy of the media and multiplication of the elastica interna were observed in most of the vessels showing high CSIM values in any of the groups. Particularly, hypertrophy of media was closely associated with elevation of the CSIM values. There were no findings of emboli, arteritis, myocarditis and vascular necrosis in our cases. The fact that atherosclerosis was frequently observed in the intima of epicardial coronary artery, whereas changes in the intima were minimal in the intramural branches has been already reported ${ }^{6-10}$ That muscular hypertrophy of the media is the major cause of stenosis of the intramural branches has also been observed in SHR rats, an animal model of essential hypertension.11 In the present study, however, vessels with hypertrophy of media were observed not only in the Hypertension group but also in the Infarct-H and Valvular-A groups in which left ventricular pressure had been elevated. The incidence of hypertrophy of media was significantly higher in these three groups than the others with no elevation of left ventricular pressure. These findings indicated that hypertrophy of the media is closely related to left ventricular pressure or intramural pressure of the ventricle.

In the heart in vivo, vascular tension is determined by resting tension, which is the function of the inner diameter of the vessel, and active vascular tension. ${ }^{16}$ Since, even with maximal vascular dilatations which is the similar state to the stretched vessels after postmortem coronary angiography, decreased inner diameter caused by the wall thickening of the intramural branches elicits increased vascular resistance by Poiseuille's law, blood flow through the myocadial wall is assumed to decrease. Furthermore, remarkable reduction of blood flow may be induced with slight increase of active vascular tension in the stenotic intramural branches.

It is generally observed that in the arterial walls such as in the carotid artery, mesenteric artery and renal artery, hypertrophy of the media occurs with elevation of intravascular pressure, leading to stenosis of the arterial lumen. As the mechanism of hypertrophy of the media, adaptive response of the vascular wall to elevation of intravascular pressure ${ }^{17}$ and vasoconstrictive reaction ${ }^{18}$ have been postulated to be an essential factor as well as high intravascular pressure. Intramural coronary branches, however, are different from other arteries in that, in the former, extravascular pressure is extremely high. The results of the present study indicate that the wall thickening of intramural coronary branches is more closely related to intramural pressure than to intravascular pressure. This suggests that intramural pressure in the myocardium which compresss from outside of the vessel synchronizing with cadiac cycle plays a more important role than intravascular pressure in the pathogenesis of stenosis in the intramural coronary branches.

\section{Acknowledgement}

The author wishes to express sincere appreciation to Professor Hisashi Fukuzaki, the First Department of Internal Medicine, Kobe University School of Medicine, for his invaluable guidance and critical review of the manuscript, Dr. Kiichiro Tanemoto, Deputy Director, Kobe Rosai Hospital and Dr. Goro Tsuchiya, Deputy Director, Mitsubishi Kobe Hospital, for their guidance in conducting the present study, Dr. Satoru Matsuura, Director, Hyogo Prefectural Awaji Hospital, for his guidance on pathological studies and the colleagues of Kobe Rosai Hospital for their co-operation and assistance.

\section{REFERENCES}

1. KAJIYA F, HOUKI N, NANTO S: A genesis and model of phasic coronary blood flow. In Coronary Circulation, ed. by TAKENAKA $\mathrm{N}$, Asakura shoten, Tokyo, 1978, p 62 (in Japąnese)

2. HOFFMAN JIE, BUCKBERG GD: Transmural variations in myocardial perfusion. In Progress in Cardiology, ed. by YU PN and GOODWIN JF, Lee and Febiger, Philadelphia, 1976, p 37

3. JAMES TN: Pathology of small coronary arteries. Amer J Cardiol 20: 679, 1967 
4. GROSS L, EPSTEIN EZ, KUGEL MA: Histology of the coronary arteries and their branches in the human heart. Amer J Path 10: 253, 1934

5. SAPHIR O, OHRINGER L, WONG R: Changes in the intramural coronary branches in coronary arteriosclerosis. AMA Arch Path 62: 159, 1956

6. DONOMAE I, MATSUMOTO Y, KOKUBU T, KOIDE R, KOBAYASHI R, IKEGAMI H, UEDA E, FUJIWARA T, FUJIMOTO S: Pathological studies of coronary athelosclerosis: Especially of sclerosis of intramural coronary arteries. Jap Heart $J$ 3: 423, 1962

7. HATANI $\mathrm{H}$ : Angiographic, histometric and histopathological studies on the intramural coronary arteries in the infarcted heart. Acta Path Jap 27: 511,1977

8. RODRIGUEZ FL, REINER L: A new method of dissection of the heart. AMA Arch Path 63: 160, 1957

9. VAN CITTERS R, WAGNER BM, RUSHMER RF: Architecture of small arteries during vasoconstriction. Circ Res 10: 668, 1962

10. SHOZAWA $\mathrm{T}$ : A statistical analysis of coronary stenosis based on 1150 autopsy cases. Acta Gerontol Jap 31: 121, 1959 (in Japanese)

11. SAWAWATARI S, YAMADA K, TOMINAGA K, EIJIMA I, SHIGIYA R: Coronary circulation in hypertensive hearts. J Jap Coll Angiol 13: 339, 1973 (in Japanese)

12. OOSAWA K: Clinicopathological studies on the coronary circulation with postmortem coronary angiography. Gumma J Med Sc 16: 146, 1967

13. PARKER FB Jr, NEVILLE JF Jr, HANSON EL, WEBB WR: Retrograde and antegrade pressure and flows in preinfarction syndrome. Circulation 49 and 50 (Supple II): 122, 1974

14. STEIN PD, MARZILLI M, SABBAH HN, LEE T: Systolic and diastolic pressure gradients within the left ventricular wall. Amer J Physiol 238: H 652 , 1980

15. BAIRD RJ, MANKTELOW RT, SHAR PA, AMELI FM: Intramyocardial pressure. A study of its regional variations and its relationship to intraventricular pressure. $J$ Thorac Cardiovasc Surg 59: 810,1970

16. HOUKI N, INOUE M, KAJIYA F, KATO T, INADA $\mathrm{H}$, FUKUSHIMA $\mathrm{M}$, NANTO S, TSUJIOKA K, ABE H: A simulation study of coronary circulatory system. A theoretical analysis of intramyocardial flow distribution mechanism. Jap Circ J 41: 1279, 1977

17. LIEBOW AA: Situations which lead to changes in vascular patterns. In Handbook of Physiology, Sec 2, Circulation, Vol 2, ed. by HAMILTON WF, American Physiological Society, Washinton D.C., 1963 , p 1251

18. SHORT DS, THOMSON AD: The arteries of the small intestine in systemic hypertension. $J$ Path Bact 78: 321, 1959 